\title{
The Isothermal Cure Kinetic of Epoxy/Amine System Analyzed by Phase Change Theory
}

\author{
Sang-Wook Kim, ${ }^{\dagger}$ Man-Geng LU, AND Mi-Ja SHIm* \\ Department of Chemical Engineering, The University of Seoul, Seoul 130-743, Korea \\ * Department of Life Science, The University of Seoul, Seoul 130-743, Korea
}

(Received April 18, 1997)

\begin{abstract}
The cure behavior of diglycidylether of bisphenol A (DGEBA)-methylene dianiline (MDA) and DGEBAcarboxyl-terminated butadiene-acrylonitrile copolymer (CTBN)-MDA systems was investigated at the different temperatures. A detailed analysis of the cure process has been made utilizing the Avrami expression. The kinetic parameters were obtained and were in good agreement with the results from other analytical methods. In some cases, the even more satisfactory explanations can be given by the phase change theory. Both of epoxy resin systems have a similar cure mechanism, but the CTBN composition can induce the faster heterogeneous nucleation and exhibits the shorter half-life. The experimental kinetic results from Avrami theory may present combined effect.

KEY WORDS Avrami Equation/Phase Change / Cure Kinetics / Epoxy Resin / Differential Scanning Calorimetry /
\end{abstract}

Epoxy resins now are widely used as the matrix of so-called advanced composite materials. The properties of ultimate material very much depend on its process and cure conditions. Even though many experimental techniques and studies relating to epoxy cure reactions have been reported in the literature with emphasis on the chemical, physical, and mechanical property changes with time, ${ }^{1,2}$ there are often conflicts in the kinetic parameters. Moreover, data can be interpreted by different methods to produce different results. These problems arise primarily from the assumptions used in the calculations and the negligence of the physical phenomena likely to take place in the system. Therefore, it is necessary to understand the fundamental principles that govern the process. ${ }^{3,4}$

The three-dimensional polymerization of multifunctional monomers or oligomers, traditionally, was considered an available avenue for producing highly crosslinked polymer networks with desired structure and properties. However, extensive studies have shown that the polymerization is not limited to chemical crosslinking and that the relationship between initial oligomer and resulting polymer structure and properties is not so straightforward as it might appear. ${ }^{5}$ In many instances this process is accompanied by micro-phase separation, vitrification of reaction medium, and heterogeneous polymer network formation. These physical and structural changes in turn often significantly affect both polymerization kinetics and the structure and properties of the resulting polymers. ${ }^{6,7}$ Therefore, it is rather significant to find a new kinetic model relative to all of these factors and further understanding the cure process of epoxy resin systems.

In general, kinetic expressions may be phenomenological or mechanistic. Phenomenological models relate to the main features of the reaction kinetics and do not take into account individual reactions, whereas mechanistic models are obtained from balances of species involved in the reaction. In our opinion, the phenomenological approach prefers in this study because the cure process is so complex that it is very difficult to identify each and every reaction, especially, the mechanistic model will not be valid for the later stages of cure where the reaction mechanism is diffusion-controlled.

Avrami theory based on the phase change was most often used to describe polymer crystallization kinetics. In a broad sense, crystallization can be considered as a physical form of crosslinking. In some aspects the behavior of the amorphous crosslinked polymers at a higher degree of crosslinking is similar to that of at a higher degree of crystallinity. So it is also possible to predict the cure process of epoxy resin using Avrami expression. Meanwhile, many molecular aggregates or high molecular weight particles have been observed when an infinite network was formed as a result of crosslinking during cure reactions.

However, there is little attention held in this field. Pollard predicted the gel point of multifunctional epoxyamine system at which the deviation of experiment from theory occurred for the degree of cure. ${ }^{8}$ The theory also has been used to predict nonisothermal behavior of diglycidylether of bisphenol A (DGEBA)-methylene dianiline (MDA) system from isothermal data, through Avrami equation. ${ }^{9}$ However, all those works were based on the assumption of $n$-th order reaction rate and a negligible reaction between secondary amine and epoxy group. It is not real in most cases so that a perfect match of the theoretical predictions with the experimental data can not be achieved.

In our laboratory, the cure kinetic behavior of epoxy systems and their composites has been conducted systematically. We now focus on the phenomenological model and the fundamental kinetic results and analysis will be given as the preliminary experiments using differential scanning calorimetry (DSC). In this research, isothermal experiments were performed for DGEBAMDA and DGEBA-carboxyl-terminated butadiene-acrylonitrile copolymer (CTBN)-MDA systems. The cure kinetic parameters and a suggested mechanism based on Avrami theory have been given.

\footnotetext{
† To whom all correspondence should be addressed.
} 


\section{ANALYSIS OF DATA}

The DSC thermograms are analyzed based on the following assumptions: 1) The area under the curves is proportional to the extent of reaction. 2) The heat released at any time (temperature) is proportional to the number of mols reacted at that time. Also, the extent of reaction during the mixing of the epoxy resin and the hardener is neglected. The reaction was considered complete when the rate curve levelled off to a baseline. The total area under the exotherm curve based on the extrapolated baseline at the end of the reaction gives the isothermal heat of cure.

In the standard isothermal method, the DSC curves were constructed by integrating the area under the exothermic peak according to the following equation:

$$
\alpha_{\mathrm{t}}=\frac{\int_{0}^{t}\left(\frac{\mathrm{d} H_{\mathrm{t}}}{\mathrm{d} t}\right) \mathrm{d} t}{\int_{0}^{\infty}\left(\frac{\mathrm{d} H_{\mathrm{t}}}{\mathrm{d} t}\right) \mathrm{d} t}
$$

where $\mathrm{d} H_{\mathrm{t}} / \mathrm{d} t$ is the rate of evolution of heat, $t$, is the time, $\alpha_{t}$ is the extent of cure at time $t$. In the Miller method the $\alpha_{\mathrm{t}}$ value is given by ${ }^{10}$ :

$$
\alpha_{\mathrm{t}}=\frac{\Delta H_{\mathrm{t}}}{\Delta H_{0}}
$$

where $\Delta H_{\mathrm{t}}$ is the heat generated up to time $t ; \Delta H_{0}$ is the total heat of complete cure.

The isotherms obtained were analyzed by means of the simple Avrami equation:

$$
\left(1-\alpha_{t}\right)=\exp \left(-k t^{n}\right)
$$

where the rate constant $k$ and Avrami exponent $n$ were calculated either from a plot according the relation

$$
\log [-\ln (1-\alpha)]=\log k+n \log t
$$

or directly from the relations:

$$
k=\frac{\ln 2}{t_{0.5}^{n}}
$$

where $t_{0.5}$ is defined as the time at which the extent of cure is $50 \%$ complete.

\section{EXPERIMENTAL}

The epoxy resin systems used in this study consist of a commercial DGEBA (Shell Chemicals, Epon 828), 4,4'-MDA (Fluka Chemie Co.) and a low molecular weight CTBN (26\%) (Kukdo Chemical Co.). These materials were used as supplied without further purification. The epoxide equivalent weight of the resin was taken as 184 and the concentration of CTBN is $10 \%$.

The resin was cured employing stoichiometric quantities of diamine. The two systems are referred as DGEBAMDA and DGEBA-CTBN-MDA, respectively. The epoxy resin was mixed with CTBN and melted MDA under good stirring about $80^{\circ} \mathrm{C}$ for $3-5 \mathrm{~min}$, then cooled rapidly to room temperature. The resulted material was kept in a freezer at $-20^{\circ} \mathrm{C}$ and under desiccation prior to use. Caution had to be taken to avoid over- heating the materials, especially for the epoxy system containing CTBN oligomer in which the fast cure reaction will take place at the higher temperature.

Samples consisting of 3-6 mg mixture are placed in aluminum DSC pans and run on a SEIKO I-5000 series. In the isothermal scans, the test samples are heated to the test temperature at $100^{\circ} \mathrm{C} \mathrm{min}^{-1}$ at five temperature between $80-120^{\circ} \mathrm{C}$. A steady isothermal baseline is established at the present curing temperature using two empty sample pans. Temperature equilibrium at the sample and reference holder is obtained in less than $2 \mathrm{~min}$ after the sample was introduced in the cell. A time record of the rate at which heat was generated was made until the curve levelled off to the baseline. The total heat of reaction, $\Delta H_{0}$ in eq 2 , was obtained by scanning the sample from 20 to $250^{\circ} \mathrm{C}$ at heating rate of $10^{\circ} \mathrm{C} \mathrm{min}^{-1}$. The carrier gas is nitrogen at a flow rate of $50 \mathrm{ml} \mathrm{min}^{-1}$.

\section{RESULTS AND DISCUSSION}

Typical DSC isotherm curves for DGEBA-MDA and DGEBA-CTBN-MDA systems were shown in Figure 1. The curves indicate the CTBN composition produces acceleration in the early stage of the cure reaction. The degree of cure as a function of curing time was plotted in Figures 2 and 3 for two systems. All of the curves have a typical shape of epoxy cure behavior, indicating that similar curing mechanism is occurring, and state clearly that the prereaction occurred during the mixing of epoxy resin and the hardener (less than $5 \mathrm{~min}$ ) is negligible. It is worthy to point out in here, in calorimetric measurement, the sample was simulated by a nominal high heating rate prior to the isothermal cure period. The actual temperature may temporarily overshoot the present isothermal temperature, and it requires some time to gain temperature equilibrium although the extent of non-isothermal prereaction is only small fraction of $\Delta H_{0}$. The exact period is a function of instrumental design and the time constant of the individual apparatus (less than $2 \mathrm{~min}$ in here). Because of this, it is difficult to define the initiation time, $t=0$. In this present work, the extrapolated peak onset time (intersecting point of tangent at the point of inflection/baseline) was considered as the initiation time for isothermal part of experiment by assuming the curing reaction only begins at this point. The degree of conversion at the early stage of cure may be lower than the actual due to the deviation of the baseline. But the accurate values can be obtained correspondingly by correcting the area under the exotherm curve from 0 to $t$. In addition, some heat of cure will go unrecorded in the initial equilibration period, especially, for a higher curing temperature. Therefore, no reaction was conducted isothermally over $120^{\circ} \mathrm{C}$.

From these figures, we can find that there is a higher extent of cure at the given cure temperature and time for DGEBA-CTBN-MDA system. This kind of behavior has been observed by other workers. However, a perfect interpretation can not be given using the general analytical methods. Verchere and Co et al. reported the similar experimental results. ${ }^{11,12}$ The initial acceleration of cure rate was attributed to the initial hydroxy concentration and the addition catalysts such as the 


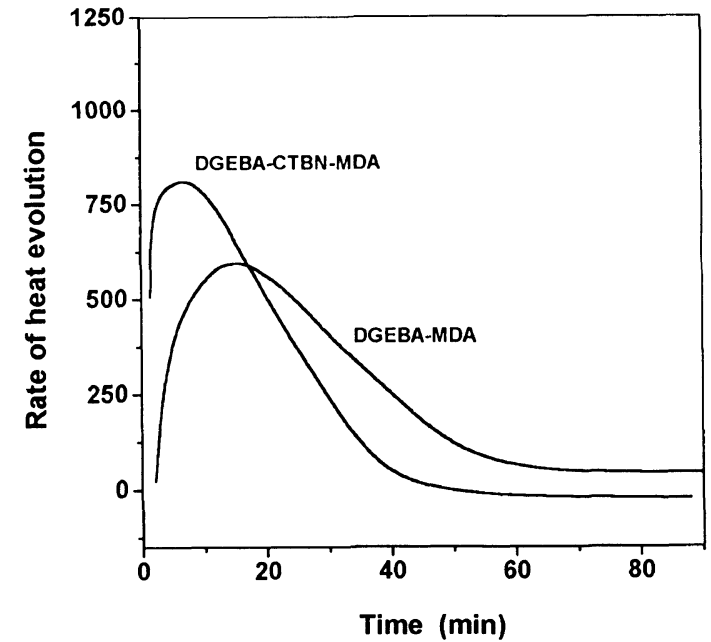

Figure 1. Typical DSC exotherm curves for DGEBA-MDA and DGEBA CTBN-MDA at $100^{\circ} \mathrm{C}$.

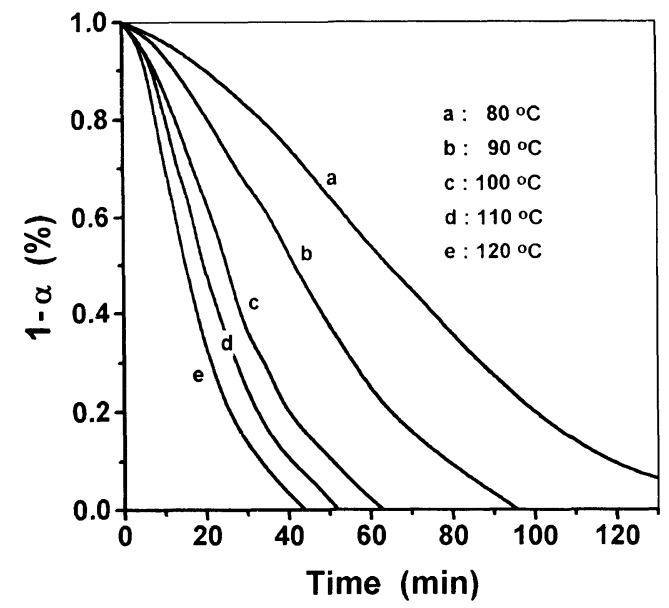

Figure 2. Cure isotherms of DGEBA-MDA at $80-120^{\circ} \mathrm{C}$.

impurities, although little hydroxy group was comprised in the CTBN. According to the Avrami theory, it is clear from Table I and from eq 3 , that in the case of large value of $k$ and small value of $n$ for DGEBA-CTBNMDA system, the fraction of cure at the early stage of cure will be larger than in the case of smaller $k$ and large $n$ for DGEBA-MDA system. This result can be attributed to the accelerating influence of CTBN which was related to the faster heterogeneous nucleation due to the incompatibility between epoxy and CTBN oligomer.

The kinetic parameters are obtained by plotting the data according to eq 4 . A plot of $\ln [-\ln (1-\alpha)] v s$. $\ln t$ yields a straight line; the slope is equal to $n$ and the intercept is equal to $\ln k$. Typical Avrami plots for two systems are given in Figures 4 and 5, respectively. A summary of $n$ and $k$ values for the cure behavior of epoxy resins is given in Table $\mathrm{I}$.

Since the Avrami exponent is contained in the rate constant, as can be seen from eq 5 , we recalculated the rate constants based on the observed cure half-time and an average values of the exponent and the results were also listed in Table I. An Arrhenius plot (Figure 6) shows a good linear correlation. The kinetic rate constant, $k$, in eq 3 determines the rates of the nucleation and growth

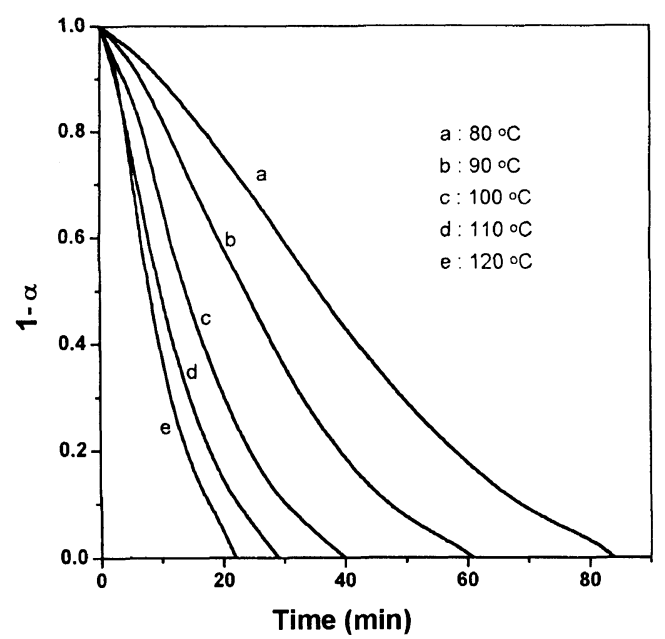

Figure 3. Cure isotherms of DGEBA-CTBN-MDA system at $80-120^{\circ} \mathrm{C}$.

process which control the cure. It is extremely sensitive to temperature (see Table I). The values are consistent with the results obtained from other analytical methods. It can be expressed as:

for epoxy-MDA system

$$
k=3.15 \times 10^{4} \exp (-64.4 / R T)
$$

for epoxy-CTBN-MDA system

$$
k=3.02 \times 10^{5} \exp (-66.8 / R T)
$$

Activation energy based on the slope of Figure 6 was obtained. As seen in Table I, $E_{\mathrm{a}}$ values are between $46 \mathrm{~kJ} \mathrm{~min}^{-1}$ for the autocatalyzed rate constant $k_{1}$ and $84 \mathrm{~kJ} \mathrm{~mol}^{-1}$ for $k_{1}{ }^{\prime}$ (catalyzed rate constant) ${ }^{3}$ and show that a balanced result is obtained from this phenomenological model. Both the activation energy $E_{\mathrm{a}}$ and the pre-exponential factor increase with the addition of CTBN. Generally, the pre-exponential factor might decrease if the added CTBN acts as a diluent. However, it was observed that the reaction was accelerated with its addition. Therefore, a diluent effect of CTBN on the cure kinetics of the epoxy resin cannot explain the above phenomenon. ${ }^{12}$ The reason of accelerating effect has been described above.

From Figures 1 and 2, the values of half-life $t_{0.5}$ at the different temperatures can be obtained. The experimental data and theoretical results calculated from eq 5 based on the obtained values of $n$ and $k$ were listed in Table I and plotted in Figure 7. The theoretical results were in good agreement with observed one. At the given temperature, the value of $t_{0.5}$ for epoxy-CTBN-MDA was shorter than that of epoxy-MDA system. Since in all cases the measured Avrami $n$ values ranged from 1.6 to 1.8 and have little change for the two systems, $t_{0.5}$ values can provide a direct means for comparing the rates of cure.

The Avrami exponent provides qualitative information on the nature of nucleation and the growth processes. Figures 4 and 5 show the $n$ values do not vary significantly with temperature. It suggests that the same cure mechanism occurs in the whole course of cure for DGEBA-MDA and DGEBA-CTBN-MDA systems. The nonintegral values of $n$ cannot be explained in 
Table I. The kinetic parameters for DGEBA-MDA and DGEBA-CTBN-MDA systems

\begin{tabular}{|c|c|c|c|c|c|c|c|c|c|c|}
\hline \multirow{3}{*}{$\begin{array}{c}\text { Temp } \\
\mathrm{K}\end{array}$} & \multicolumn{5}{|c|}{ Epoxy-MDA } & \multicolumn{5}{|c|}{ Epoxy-CTBN-MDA } \\
\hline & \multirow{2}{*}{$n$} & \multirow{2}{*}{$\bar{n}$} & $E_{\mathrm{a}}$ & \multirow{2}{*}{$k / \mathrm{s}^{-n}$} & \multirow{2}{*}{$\begin{array}{c}k \\
(\text { Calcd }) / \mathrm{s}^{-n}\end{array}$} & \multirow{2}{*}{$n$} & \multirow{2}{*}{$\bar{n}$} & $E_{\mathrm{a}}$ & \multirow{2}{*}{$k / \mathrm{s}^{-n}$} & \multirow{2}{*}{$\begin{array}{c}k \\
(\text { Calcd }) / \mathrm{s}^{-n}\end{array}$} \\
\hline & & & $\mathrm{kJ} \mathrm{mol}^{-1}$ & & & & & $\mathrm{~kJ} \mathrm{~mol}^{-1}$ & & \\
\hline 313 & - & \multirow{7}{*}{1.76} & \multirow{7}{*}{64.4} & $5.71 \times 10^{-7}$ & - & - & \multirow{7}{*}{1.64} & \multirow{7}{*}{66.8} & $2.10 \times 10^{-6}$ & - \\
\hline 353 & 1.75 & & & $9.45 \times 10^{-6}$ & $7.58 \times 10^{-6}$ & 1.64 & & & $3.87 \times 10^{-5}$ & $3.29 \times 10^{-5}$ \\
\hline 363 & 1.71 & & & $1.73 \times 10^{-5}$ & $1.62 \times 10^{-5}$ & 1.64 & & & $7.23 \times 10^{-5}$ & $6.33 \times 10^{-5}$ \\
\hline & & & & & & & & & & \\
\hline 373 & 1.81 & & & $3.05 \times 10^{-5}$ & $3.97 \times 10^{-5}$ & 1.64 & & & $1.31 \times 10^{-4}$ & $1.56 \times 10^{-4}$ \\
\hline 383 & 1.82 & & & $5.25 \times 10^{-5}$ & $6.23 \times 10^{-5}$ & & & & $2.30 \times 10^{-4}$ & $2.68 \times 10^{-4}$ \\
\hline 393 & 1.76 & & & $8.78 \times 10^{-5}$ & $9.21 \times 10^{-5}$ & 1.61 & & & $3.93 \times 10^{-4}$ & $3.63 \times 10^{-4}$ \\
\hline
\end{tabular}

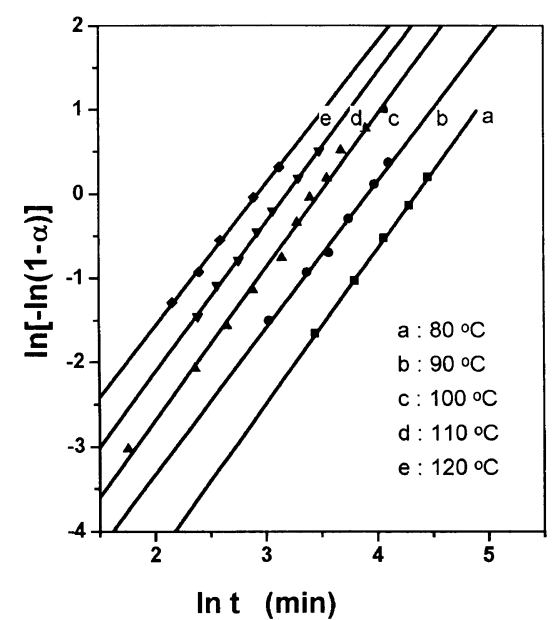

Figure 4. Avrami plot to cure behavior of DGEBA-MDA at various tempertures.

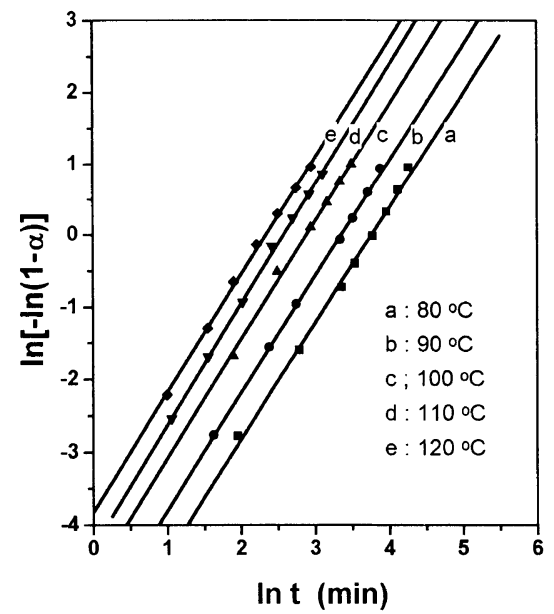

Figure 5. Avrami plot of cure behavior of DGEBA-CTBN-MDA at various temperatures.

terms of the physical assumptions made in arriving at the Avrami equation. In the formal theory of transformation kinetics, the fraction of conversion is

$$
\alpha=1-\exp \left(-g \int I_{\mathrm{v}}\left(\int u \mathrm{~d} t a u\right)^{m} \mathrm{~d} t^{\prime}\right)
$$

where $g$ is geometric factor, $I_{\mathrm{v}}$ and $u$ are the nucleation and growth rates respectively. Equation 8 is strictly valid only for interface-limiting growth, where $m$ is equal to the number of growth dimension, $n$, and is only integrated
Table II. The theoretical and experimental values of $t_{0.5}$ for both systems

\begin{tabular}{|c|c|c|c|c|}
\hline \multirow{2}{*}{$\begin{array}{c}\text { Temp } \\
{ }^{\circ} \mathrm{C}\end{array}$} & \multicolumn{2}{|c|}{ Epoxy-MDA } & \multicolumn{2}{|c|}{ Epoxy-CTBN-MDA } \\
\hline & $\begin{array}{c}t_{0.5} \min \\
(\text { exptl) }\end{array}$ & $\begin{array}{c}t_{0.5} \min \\
\text { (calcd) }\end{array}$ & $\begin{array}{c}t_{0.5} \min \\
(\text { exptl })\end{array}$ & $\begin{array}{c}t_{0.5} \min \\
\text { (calcd) }\end{array}$ \\
\hline 80 & 64.35 & 62.80 & 35.66 & 33.62 \\
\hline 90 & 41.86 & 36.38 & 23.93 & 22.53 \\
\hline 100 & 25.10 & 29.90 & 13.81 & 14.14 \\
\hline 110 & 19.42 & 21.33 & 10.05 & 10.51 \\
\hline 120 & 15.42 & 16.23 & 8.24 & 8.24 \\
\hline
\end{tabular}

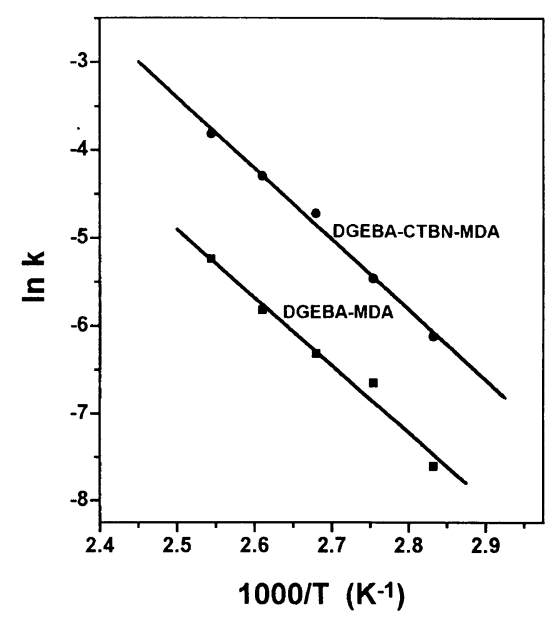

Figure 6. Rate constant dependence on temperature.

under isothermal conditions (when $I_{\mathrm{v}}$ and $u$ are assumed to be independent of time) to yield eq 3 . Therefore, fractional values of $n$ may be expected mathematically, if certain constraints are imposed such as diffusion controlled growth. The observed deviations could also be attributed to the factors such as time-dependent nucleation, variant growth rate constants, combination of homogeneous and heterogeneous nucleation.

In addition, it is difficult to precisely analyze the Avrami theory if the system was glassified wholly. However, as verified by many researchers, the transformation from a viscous liquid to a glass only begins to occur as the glass transition temperature of network becomes coincidental with the cure temperature. The limited conversion degree (the maximum degree of cure) depends on the temperature of curing. So it is essential to conduct the isothermal experiment at little higher 


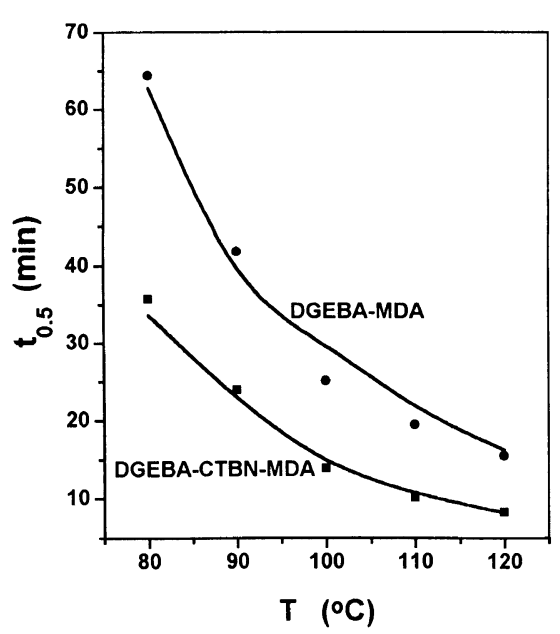

Figure 7. Relation of $t_{0.5}$ and temperature. Solid lines represent the theoretical results. Points represent the experimental data.

curing temperature to describe the whole cure process.

The reaction degree can be calculated from the constants obtained using eq 3 at 90 and $120^{\circ} \mathrm{C}$ for DGEBA-MDA and DGEBA-CTBN-MDA systems. It is accurately consistent between theoretical and experimental data. The similar behavior was observed in other temperatures. These results showed that the cure behavior of epoxy systems can be described by Avrami expression in the entire temperature range.

Since the values of Avrami exponent $n$ for both epoxy systems lie between $1-2$, in summary, the cure process may involve either homogeneous nucleation or heterogeneous nucleation with one directional growth and two directional diffusion controlled growth $(n \rightarrow 2)$. In the case of CTBN toughening epoxy resin, heterogeneous nucleation will predominantly occur on the interface forming between CTBN and resin. Homogeneous nucleation may also take place simultaneously in the prepolymer bulk. Such nonuniform nucleation could lead to complicate cure kinetics and mechanisms, and the experimental kinetic results may present a combined effect.

\section{CONCLUSIONS}

In this work, the isothermal cure behavior has been investigated for DGEBA-MDA and DGEBA-CTBNMDA systems. The results lead to the following conclusions.

The cure process for the two resin systems may involve either homogeneous or heterogeneous nucleation, and CTBN composition can induce the faster heterogeneous nucleation as a nucleating agent.

Avrami phase change theory can be used to describe the cure process of epoxy resins and can give the even more satisfactory explanation to some experimental phenomena. The kinetic parameters obtained are consistent with that from other thermal analytical methods. The kinetic results from Avrami theory may present a combined effect of all factors.

\section{REFERENCES}

1. T. Oishi and M. Fujimoto, J. Polym. Sci. Part A, 30, 1821 (1992).

2. S. Sourour and M. R. Kamal, Thermochim. Acta, 14, 41 (1976).

3. E. A. Turi, "Thermal Characterization of Polymer Materials," Academic Press, New York, N.Y., 1983.

4. B. A. Rozenberg, Adv. Polym. Sci., 72, 113 (1985).

5. A. A. Berlin and N. G. Matveyeva, Macromol. Rev., 12, 1 (1977).

6. S. Zhu and A. E. Hamielec, Makromol. Chem. Macromol. Symp., 63, 135 (1993)

7. S. B. Liu, J. L. Liu, and T. L. Yu, J. Appl. Polym. Sci., 53, 1165 (1994).

8. M. Pollard and J. L. Kardos, Polym. Eng. Sci., 27, 829 (1987).

9. M. G. Lu and S. W. Kim, to be published.

10. B. J. Miller, J. Appl. Polym. Sci., 11, 2343 (1967).

11. M. B. Ko, S. C. Kim, and W. H. Jo, Korea Polym. J., 2, 131 (1994).

12. D. Verchere, H. Sautereau, J. P. Pascault, S. M. Moschiar, C. C. Riccardi, and R. J. J. Williams, J. Appl. Polym. Sci., 41, 467 (1990). 